\title{
wissen kompakt „Implantatprothetik“
}

\author{
Für den Ersatz fehlender Zähne gibt es verschiedene Lösungen - eine davon sind implantat- \\ getragene Versorgungen. Diese haben sich als therapeutische Option etabliert und werden \\ heutzutage täglich umgesetzt. Umso wichtiger also, sich in Bezug auf die gegenwärtigen \\ Möglichkeiten und aktuellen Entwicklungen auf dem Laufenden zu halten.
}

Entsprechend widmet sich die Juni-Ausgabe von wissen kompakt dem Thema „Implantatprothetik“ und hält spannende Beiträge für Sie bereit:

\section{Digitale Konzepte in der Implantologie} PD Dr. J.-F. Güth, PD Dr. M. Stimmelmayr

In vielen Bereichen der Implantologie bieten digitale Technologien wesentliche Vorteile. Angefangen bei der digitalen Implantationsplanung und Verfahren der navigierten Chirurgie über die digitale Abformung von Einzelzahnimplantaten bis hin zum CAD/CAM-gefertigten Zahnersatz aus homogenen biokompatiblen Materialien ergeben sich viele Schnittstellen zur digitalen Zahnheilkunde. Der Beitrag geht auf verschiedene digitale Konzepte in der Implantologie ein und beleuchtet diese kritisch.

\section{Vollkeramische, implantatgetragene Versorgungen Prof. Dr. S. Hahnel}

Gerade bei aufwendigen prothetischen Restaurationen besteht ein Interesse an ästhetisch anspruchsvollen Lösungen. Vollkeramische Versorgungen können diesem Wunsch in besonderem Maße entsprechen, weswegen sie zunehmend auch als implantatgetragener Zahnersatz eingegliedert werden. Das Ziel des Beitrags besteht darin, die wissenschaftliche Datenlage für die vollkeramische Versorgung von Implantaten darzulegen und zu diskutieren sowie konkrete Handlungsanweisungen für die zahnärztliche Praxis zu entwickeln.

\section{Implantatgetragene Doppelkronen \\ Dr. F. S. Schwindling, U.K. Deisenhofer, \\ Prof. Dr. P. Rammelsberg}

Der Einsatz von Doppelkronen erlaubt neben der Anfertigung konventionellen Zahnersatzes auch die Herstellung implantatgetragener Restaurationen sowie die Kombination von Restzähnen und Implantaten. Es lassen sich starr abgestützte und resilient gelagerte Doppelkronenarten unterscheiden. Der Beitrag thematisiert die Auswahl an Doppelkronen, beschreibt den Behandlungsablauf und beleuchtet kritisch Überlebensraten sowie Schwachstellen.

\section{Stege und solitäre präfabrizierte Halteelemente PD Dr. S. Rinke, M.Sc., M.Sc.}

Abnehmbare Suprakonstruktionen können im zahnlosen Kiefer auf einer unterschiedlichen Anzahl von Implantaten mit

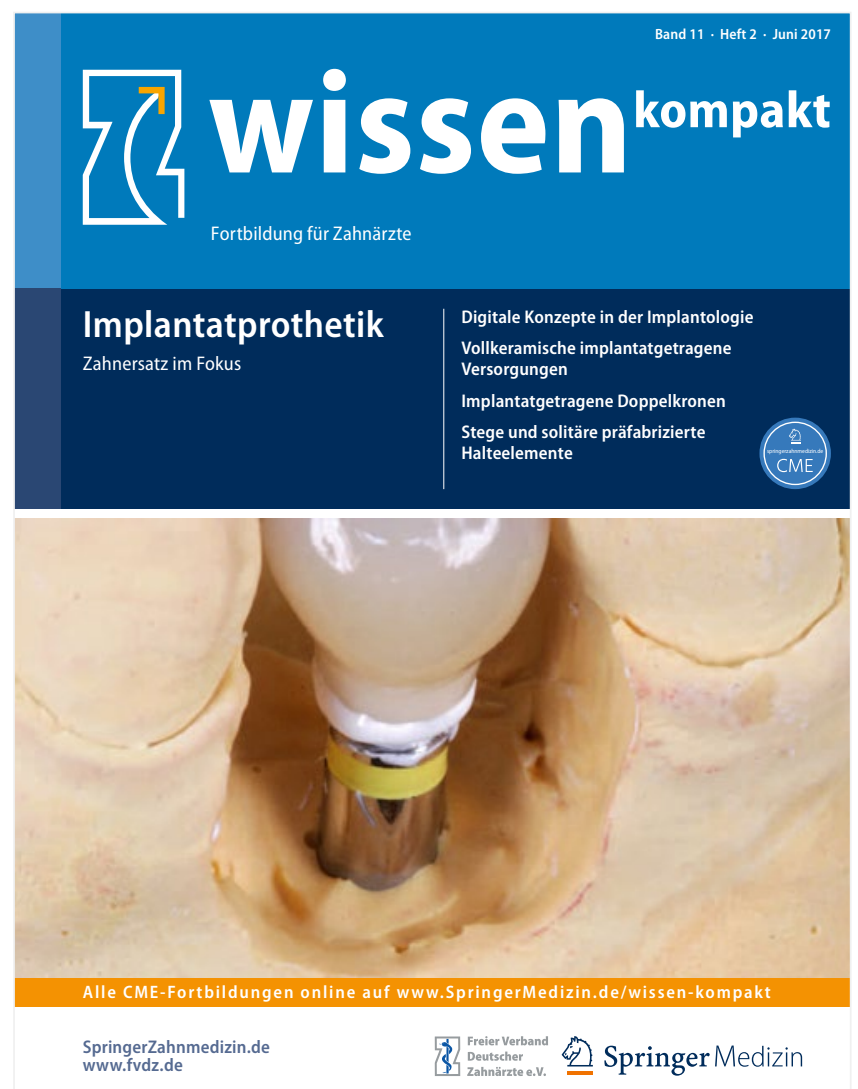

einer Vielzahl verschiedenartiger Verankerungselemente ausgeführt werden. Stege und solitäre präfabrizierte Halteelemente werden international am häufigsten zur Verankerung verwendet. Die Auswahl des Verbindungselements muss für jeden Patienten individuell aufgrund diverser Kriterien getroffen werden. Der Beitrag gibt einen Überblick über aktuell vorliegende Erkenntnisse zu implantatgetragenen und -retinierten Deckprothesen unter Verwendung von Stegen und präfabrizierten solitären Verankerungselementen.

Die aktuelle Online-Ausgabe von wissen kompakt finden Sie unter SpringerMedizin.de/wissen-kompakt 